Accelerated Article

\title{
Model Independent Determination of Colloidal Silica Size Distributions via Analytical Ultracentrifugation
}

Karel L. Planken, Bonny W. M. Kuipers, and Albert P. Philipse

Anal. Chem., 2008, 80 (23), 8871-8879 • Publication Date (Web): 25 October 2008

Downloaded from http://pubs.acs.org on December 4, 2008

\section{More About This Article}

Additional resources and features associated with this article are available within the HTML version:

- Supporting Information

- $\quad$ Access to high resolution figures

- $\quad$ Links to articles and content related to this article

- Copyright permission to reproduce figures and/or text from this article

\author{
View the Full Text HTML
}




\title{
Model Independent Determination of Colloidal Silica Size Distributions via Analytical Ultracentrifugation
}

\author{
Karel L. Planken, Bonny W. M. Kuipers, and Albert P. Philipse* \\ Van 't Hoff Laboratory for Physical and Colloid Chemistry, Debye Institute, Utrecht University, Padualaan 8, \\ $3584 \mathrm{CH}$ Utrecht, The Netherlands
}

We report a method to determine the particle size distribution of small colloidal silica spheres via analytical ultracentrifugation and show that the average particle size, variance, standard deviation, and relative polydispersity can be obtained from a single sedimentation velocity (SV) analytical ultracentrifugation (AUC) experiment. The particle size distribution (psd) from the enhanced van HoldeWeischet (vHW) analysis accounts for the dynamic light scattering results quite well. In addition, the vHW psd equals the psd from a continuous distribution of sedimentation coefficients analysis where whole sedimentation velocity boundaries are fitted. The SV AUC interference optical data also yield the specific particle volume such that distributions of sedimentation coefficients for colloidal spheres can be converted directly to particle size distributions. Our results show that SV AUC experiments may yield a quantitative particle size distribution without a priori knowledge of the particle size and the shape of the size distribution.

Synthetic macromolecules as well as colloids are always to a certain degree heterogeneous with respect to size and shape.

For the size and shape determination, as well as the overall particle size distribution (psd), several techniques are available ${ }^{1-3}$ such as transmission electron microscopy (TEM, or variations on this technique such as scanning electron microscopy and cryo-

* To whom correspondence should be addressed. E-mail: science.secr.fcc@uu.nl. Fax: +31-30-253 3870 .

(1) Philipse, A. P. In Fundamentals of Interface and Colloid Science; Lyklema, J., Ed.; Elsevier Academic Press: New York, 2005; Vol. IV, pp 2.1-2.71.

(2) Cölfen, H.; Schnablegger, H.; Fischer, A.; Jentoft, F. C.; Weinberg, G.; Schlögl, R. Langmuir 2002, 18, 3500-3509.

(3) Cölfen, H.; Völkel, A.; Eda, S.; Kobold, U.; Kaufmann, J.; Puhlmann, A.; Göltner, C.; Wachernig, H. Langmuir 2002, 18, 7623-7628.
TEM) and atomic force microscopy (AFM). These microscopic techniques, except cryo-TEM, ${ }^{4,5}$ do not image in situ. Drying of TEM samples may lead to particle shrinkage and distortion of particle structures, including aggregation due to capillary forces. Moreover, the electron beam may seriously damage the colloids via the melting and sintering of nanoparticles, as is the case for the small silica particles that are subject of the present study. Even if TEM images are fairly representative for the colloids in solution, many counts are needed for reliable statistics. For example, in the case of a polydispersity around $30 \%$, typically at least 1000 counts are needed for a representative size distribution (see the case studies for various colloids in ref 1 ).

Techniques that sample colloids in situ are static light scattering (SLS) and dynamic light scattering ${ }^{6}$ (DLS). For sufficiently narrow size distributions, SLS data in the Guinier region can be analyzed with a momentum-expansion, to obtain an effective radius that is independent of the detailed shape of the distribution function. ${ }^{1}$ For a broad size distribution, however, it is hardly possible to extract in any a priori fashion reliable distribution parameters from static light scattering profiles. DLS may be conducted on polydisperse colloids for which the particle shape is known, to obtain an apparent average particle size and standard deviation. The shape of the particle size distribution from DLS, however, may be highly questionable because of the ill-conditioned inversion problem encountered in DLS. ${ }^{6}$ Since the scattered light intensity scales with the particle volume squared, small particles in the presence of relatively large particles are difficult to detect with DLS. The scaling of the scattered light intensity also implies

(4) Klokkenburg, M.; Vonk, C.; Claesson, E. M.; Meeldijk, J. D.; Erné, B. H.; Philipse, A. P. J. Am. Chem. Soc. 2004, 126, 16706-16707.

(5) Klokkenburg, M.; Dullens, R. P. A.; Kegel, W. K.; Erné, B. H.; Philipse, A. P. Phys. Rev. Lett. 2006, 96, 0372031-0372034.

(6) Brown, W. Dynamic light scattering: the method and some applications; Clarendon Press: Gloucestershire, U.K., 1993. 

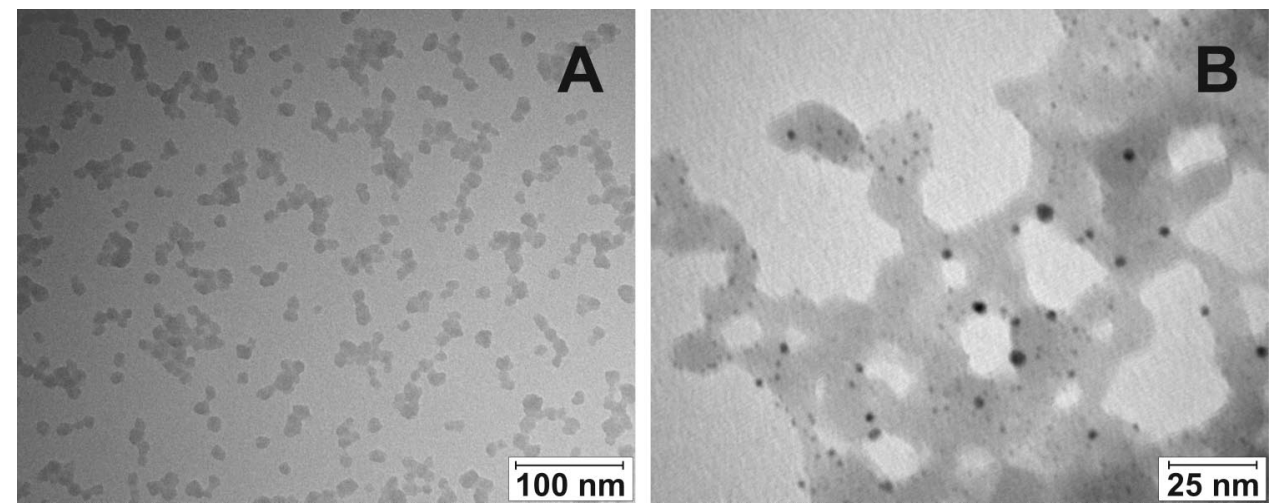

Figure 1. TEM micrographs of bare Bindzil 30/360 particles, image A, and with gold nanoparticles attached (for details, see ref 22), image $B$. Clearly, the small amorphous silica nanoparticles melt in the electron beam and fuse into big liquidlike silica blobs. Since no individual particles are observed, the size and shape of such small silica particles cannot be determined with any reasonable precision from TEM micrographs.

that DLS experiments are strongly affected if dust contaminated samples are employed.

Remarkably, analytical ultracentrifugation was initially developed in the 1920 s by Svedberg and co-workers ${ }^{7-9}$ to study colloidal gold particle size distributions. Despite the possibilities of AUC, the method has hardly been applied to analyze the psd of inorganic colloids and nanoparticles. This is partly due to the fact that AUC is primarily applied in biochemistry and cell biology and much less in the field of inorganic colloids and nanoparticles (see, however, refs 10-15). In addition, the potentiality of AUC (including the improved data analysis explained later) to quantify a psd of nanoparticles may have been overlooked or underestimated due to the absence of a convincing case study. The aim of this contribution is to provide such a study and to demonstrate that sedimentation velocity (SV) AUC may quantitatively yield a psd, without a priori information about the range and shape of this distribution.

For this case study, we choose an aqueous dispersion of amorphous silica nanoparticles for several reasons. Such silica sols are readily available, being produced and applied commercially on a large scale. ${ }^{1}$ Moreover, the small silica colloids have precisely the features that makes their psd difficult to obtain via other techniques than AUC: their shape is uncertain and they easily melt in an electron beam (Figure 1).

Our approach in more detail is as follows. The sedimentation coefficient range from the enhanced van Holde-Weischet (vHW) analysis ${ }^{16,17}$ method (UltraScan) ${ }^{18}$ is used to initialize and also to restrict the search space for the following 2-dimensional spectrum

(7) Svedberg, T.; Nichols, J. B. J. Am. Chem. Soc. 1923, 45, 2910-2917.

(8) Svedberg, T.; Rinde, H. J. Am. Chem. Soc. 1924, 46, 2677-2693.

(9) Svedberg, T.; Pedersen, K. O. The Ultracentrifuge; Clarendon Press: Gloucestershire, U.K., 1940.

(10) Mächtle, W.; Börger, L. Analytical Ultracentrifugation of Polymers and Nanoparticles; Springer Laboratory; Springer Verlag: Berlin and Heidelberg, 2006.

(11) Mächtle, W. Biophys. J. 1999, 76, 1080-1091.

(12) Cölfen, H. In Analytical ultracentrifugation: techniques and methods; Scott, D. J. D., Harding, S. E., Rowe, A. J., Eds.; Royal Society of Chemistry: London, 2005; pp, xxiii, 587.

(13) Roy, S.; Planken, K. L.; Kim, R.; v. d. Mandele, D.; Kegel, W. K. Inorg. Chem. 2007, 46, 8469-8471.

(14) Cölfen, H. Polym. News 2004, 29, 101-116.

(15) Müller, H. G. Prog. Colloid Polym. Sci. 2004, 127, 9-13.

(16) van Holde, K. E.; Weischet, W. O. Biopolymers 1978, 17, 1387-1403.

(17) Demeler, B.; Van Holde, K. E. Anal. Biochem. 2004, 335, 279-288. analysis (2DSA ${ }^{19}$ implemented in UltraScan $\left.{ }^{18}\right)$. The 2DSA allows the determination of the sedimentation and frictional ratio distributions simultaneously. For the latter analysis, the frictional ratio range of 1-4 is taken, which covers a large spectrum of shapes, i.e. from a spherical to a highly extended geomety. If the particles are all spherical then the sedimentation coefficient distribution from the enhanced van Holde-Weischet (vHW) analysis (time-invariant noise determined with the 2DSA is subtracted from the experimental data), which is corrected for diffusion, is transformed to a psd. We also show how the specific particle volume, required for the transformation of a sedimentation coefficient distribution into a radius distribution, can be obtained from the same interference optical data without conducting any additional experiments. This AUC SV procedure without any prior knowledge of the range of sedimentation coefficients or, equivalently, particle sizes and particle shapes, allows the determination of the average size and the overall psd in a model independent manner. The results obtained from the enhanced vHW analysis are compared with the results of a continuous sedimentation coefficients distribution analysis method ${ }^{20,21}$ and with results from experimental dynamic light scattering (DLS) data which were fitted using, among others, the psd as obtained from the SV AUC experiment.

\section{BACKGROUND}

Sedimentation Velocity. A macromolecule or colloid in a centrifugal field is subjected to a centrifugal force, a friction force, and a buoyant force. A balance of these forces is almost instantaneously achieved, from which an expression for the sedimentation coefficient at infinite dilution $\left(s^{\circ}\right)$, i.e. the ratio of the sedimentation velocity and acceleration, is easily obtained:

$$
s^{\circ}=\frac{V_{\mathrm{p}}\left(\rho_{\mathrm{p}}-\rho_{\mathrm{s}}^{\circ}\right)}{f^{\circ}}=\frac{m_{\mathrm{p}}\left(1-\bar{\nu}_{\mathrm{p}} \rho_{\mathrm{s}}^{\circ}\right)}{f^{\circ}}
$$

(18) Demeler, B. In Analytical ultracentrifugation: techniques and methods; Scott, D. J. D., Harding, S. E., Rowe, A. J., Eds.; Royal Society of Chemistry: London, 2005; pp 210-230.

(19) Brookes, E.; Demeler, B. Colloid Polym. Sci. 2008, 286, 139-148.

(20) Schuck, P. Biophys. J. 2000, 78, 1606-1619.

(21) Schuck, P. In Analytical ultracentrifugation: techniques and methods; Scott, D. J. D., Harding, S. E., Rowe, A. J., Eds.; Royal Society of Chemistry: London, 2005; pp 26-49.

(22) Claesson, E. M.; Philipse, A. P. Colloids Surf. A: Physicochem. Eng. Aspects 2007, 297, 46-54. 

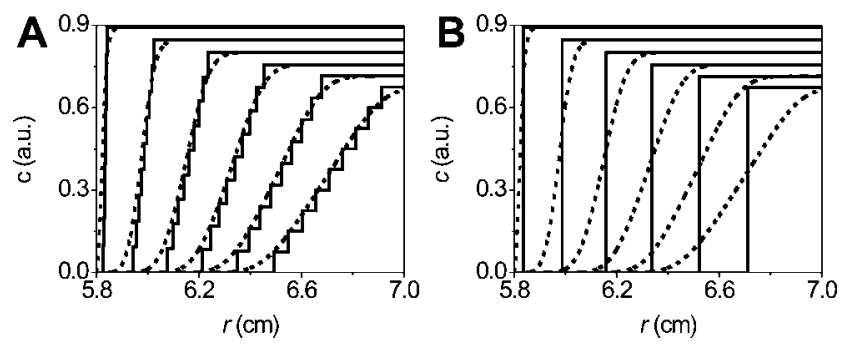

Figure 2. Simulated sedimentation velocity scans, with (- - -) ASTFEM $^{25}$ implemented in UltraScan) ${ }^{18}$ and without $(-)$ diffusion, accounting for rotor acceleration. In this case, the solution contains equal amounts of nine different solutes (graph A). The simultaneous sedimentation and diffusion of solutes gives rise to sigmoidal boundaries. Without prior knowledge of the composition of the sample, the boundaries may also correspond to the sedimentation and diffusion of a single component (graph B). Therefore, without further analysis to determine diffusion deconvoluted sedimentation coefficients, it is impossible to differentiate boundary broadening due to heterogeneity from diffusional boundary spreading.

Here, $f$ is the frictional coefficient of a sphere with radius $a_{\mathrm{h}}$ according to Stokes' equation: ${ }^{23}$

$$
f_{\text {sphere }}{ }^{\circ}=6 \pi \eta_{\mathrm{s}}{ }^{\circ} a_{\mathrm{h}}
$$

Furthermore, $V_{\mathrm{p}}$ is the particle volume, $\rho_{\mathrm{p}}$ and $\rho_{\mathrm{s}}{ }^{\circ}$ are, respectively, the particle and solvent densities, and $\eta_{\mathrm{s}}{ }^{\circ}$ is the solvent visosity. The radius of the particle can be obtained from eq 1 assuming that the particle mass $m_{\mathrm{p}}$ and its partial specific volume $\bar{\nu}_{\mathrm{p}}$, or, equivalently, the particle volume and its density are known. If the bare particle radius $a_{\mathrm{b}}$ equals the hydrodynamic radius $a_{\mathrm{h}}$, then eq 1 reduces to the following:

$$
s^{\circ}=\frac{2}{9} a_{\mathrm{b}}{ }^{2} \frac{\left(\rho_{\mathrm{p}}-\rho_{\mathrm{s}}^{\circ}\right)}{\eta_{\mathrm{s}}^{\circ}}
$$

For monodisperse noninteracting spheres, the size determination, from eq 3 , would be straightforward if diffusional sedimentation velocity boundary spreading would be absent provided, of course, that the particle density is known.

Generally, sedimentation and diffusion are superimposed in an analytical ultracentrifugation (AUC) sedimentation velocity experiment, ${ }^{24}$ as illustrated in Figure 2 by the simulated sedimentation velocity boundaries for a solution containing nine different solutes using the adaptive space-time finite element (ASTFEM) method $^{25}$ simulation module in UltraScan ${ }^{18}$ Here, we simulated nine different solutes to obtain smooth sedimentation boundaries, i.e. the different solutes are not resolved during a sedimentation velocity run due to diffusion. If a sample with fewer solutes with more disparate molecular weights would have been simulated, then the resulting sedimentation velocity boundaries look like a staircase.

Since particle size and shape heterogeneity also lead to sedimentation velocity boundary broadening, a model independent analysis, i.e. without any prior knowledge of the range of sedimentation coefficients and particle shapes, is required to

(23) Stokes, G. G. Trans. Cambridge Phil. Soc. 1850, 9, 8-106.

(24) Schachman, H. K. Ultracentrifugation in Biochemistry; Academic Press: New York and London, 1959.

(25) Cao, W.; Demeler, B. Biophys. J. 2005, 89, 1589-1602. obtain diffusion deconvoluted sedimentation coefficients to eventually determine a particle size distribution (psd). Such an analysis is the enhanced van Holde-Weischet analysis ${ }^{17}$ (vHW, Ultra$\left.\operatorname{Scan}^{18}\right)$.

The vHW analysis is based on the principle that transport due to sedimentation is proportional to time, whereas diffusional displacement is proportional to the square-root of time. Briefly, for a vHW analysis, the $m$ sedimentation velocity boundaries are each discretized in $n$ equally sized fractions between the lower (baseline) and upper stable plateaus (horizontal regions). For each boundary division $j$, at radial position $r_{j}$ of scan $i$ recorded at time $t_{i}$, a corresponding apparent sedimentation coefficient $\left(s_{i, j}^{*}\right)$ is calculated via the following:

$$
s_{i, j}^{*}=\frac{1}{\omega^{2}\left(t_{i}-t_{0}\right)} \ln \left[\frac{r_{j}\left(t_{i}\right)}{r_{\mathrm{a}}\left(t_{0}\right)}\right]
$$

where $1 \leqslant i \leqslant m, 1 \leqslant j \leqslant n$. In eq $4, \omega$ is the angular velocity, $r_{\mathrm{a}}$ is the position of the sample meniscus, and $t_{0}$ is the start time of the SV AUC run (corrected for rotor acceleration). The apparent sedimentation coefficients are then plotted versus the inverse square-root of the corresponding scan time $t_{i}$. Diffusion corrected sedimentation coefficients are obtained by extrapolating each linear fit of apparent sedimentation coefficients for the boundary fractions $j$ to infinite time. The linear extrapolation to $t=\infty$ in the vHW analysis method is achieved via a formula based on a Faxéntype approximate solution of the Lamm equation (e.g., infinite solution column length), which introduces restrictions to the scans for analysis. The modified and so-called enhanced vHW analysis circumvents the majority of the drawbacks associated with the classic vHW method. ${ }^{16}$ For example, the enhanced vHW analysis allows inclusion of scans that have not cleared the meniscus and that lack stable lower and upper horizontal regions, in contrast to the classical vHW. See ref 17 for a detailed derivation and discussion of the enhanced vHW analysis.

In contrast to a continuous distribution of sedimentation coefficients analysis $^{20,21}(c(s))$ where the sedimentation velocity boundaries for the species under consideration are fitted with a single frictional ratio, i.e. all particles have the same shape, the vHW analysis can be used for any kind of colloids, regardless of their shape. The deconvolution of diffusion and sedimentation via the enhanced vHW analysis is of special importance for spherical particles, because the assessment of size polydispersity with this analysis is then straightforward. For other particle shapes or for particles that exhibit shape heterogeneity, an equivalent sphere radius may be introduced to assess an apparent size polydispersity.

The psd can be obtained by converting the sedimentation coefficient distribution, corrected for diffusion, via eq 1 or, for spherical particles, via eq 3 . This conversion requires the input of particle density, solvent viscosity, and solvent density. The particle density, however, can be determined via several (other) techniques, e.g. density gradient centrifugation or refractive index measurements. Here, we use a specific particle volume (equivalent to the inverse particle density) that is obtained from a single analytical ultracentrifugation sedimentation velocity run using the interference optics (Beckman Coulter Optima XL-I AUC); see Materials and Methods section. 
It should be noted that increasing the angular velocity of the AUC rotor containing a sample with a sufficiently long solution column, suppresses sedimentation boundary broadening due to diffusion and therefore enhances the sedimentation coefficient resolution. For a vHW analysis, it is therefore recommended to apply the fastest angular velocity possible. The maximum angular velocity, however, is restricted by the speed with which the scans are recorded, the degree of polydispersity, and the minimum amount of scans that need to be included in the analysis. Also, excluding early scans, where the fractionation of different sized particles is minimum and the diffusion due to the initially steep concentration gradient is maximal, improves the sedimentation coefficient resolution.

The diffusion on the other hand, can provide important shape information. For such an experiment, the angular velocity should be relatively low to obtain as much information on particle shapes as possible. Regarding the assessment of particle shape, an analysis method is available (implemented in UltraScan ${ }^{18}$ ) that allows to simultaneously determine the sedimentation coefficients and frictional ratios via a sedimentation velocity whole boundary fitting routine. This 2 -dimensional spectrum analysis (2DSA) ${ }^{19}$ was initially intended for the parameter initialization to confine the search space for the genetic algorithm optimization ${ }^{26}$ for sedimentation velocity data fitting. The 2DSA decomposes the experimental sedimentation velocity data into a sum of finite element solutions to the Lamm equation, describing the noninteracting settling particles present in solution. Because the computational effort to decompose sedimentation and diffusion can quickly become very large (typically several gigabytes of RAM are required), the implementation of parallel computing using supercomputers (clusters) facilitates this analysis method. The solution obtained, thus provides information on the distribution of sedimentation coefficients and shapes (frictional ratios). Clearly, this analysis works best if fast and slow speed sedimentation velocity experiments are combined in a global fitting routine. In principle, if accurate sedimentation and diffusion coefficients are obtained, this method allows the determination of molecular weights via the so-called Svedberg-relation.

We emphasize here that the analysis methods addressed previously, to determine the psd, hold for noninteracting macromolecules or colloids. Interparticle attractions and repulsions may have pronounced effects on the sedimentation behavior, resulting in a nonrepresentative psd.

Dynamic Light Scattering. The ensemble averaged scattering intensity measured for dilute dispersions in static light scattering experiments is the sum of the intensity scattered by individual colloids in the so-called scattering volume. The intensity scattered by a single particle is proportional to the squared scattering amplitude $B(\kappa, a)$ that depends on the particle radius $a$ and on the scattering wavevector magnitude $\kappa$. The latter quantity follows from a transformation of the scattering angle $\theta$ :

$$
\kappa=\frac{4 \pi n_{\mathrm{d}}}{\lambda_{\mathrm{o}}} \sin \frac{\theta}{2}
$$

Here, $n_{\mathrm{d}}$ is the dispersion refractive index and $\lambda_{0}$ is the wavelength

(26) Brookes, E.; Demeler, B. In Progress in Colloid and Polymer Science, Analytical Ultracentrifugation VIII; Kremer, F., Richtering, W., Wandrey, C., Cölfen, H., Eds.; Springer: New York, 2006; Vol. 131, pp 33-40. of the incident light in vacuo. The scattering amplitude $B$ scales with the particle volume, and consequently, for spherical particles, $B^{2}$ scales with $a^{6}$.

In dynamic light scattering (DLS) ${ }^{27}$ experiments, the fluctuations of the intensity $I$ due to the Brownian motion of particles is characterized by the intensity autocorrelation function (IACF) defined as follows:

$$
g_{I}=\left\langle I\left(\kappa, t_{0}\right) I\left(\kappa, t+t_{0}\right)\right\rangle
$$

In the case of monodisperse spheres, the normalized IACF appears to be $\mathrm{b}^{28}$ the following:

$$
\hat{g}_{I}=1+C \exp \left[-2 D_{0} \kappa^{2} t\right]
$$

with $D_{0}$ being the Stokes-Einstein ${ }^{23,29}$ translational diffusion coefficient that is inversely proportional to the particle radius $a$. In other words, the decay of the IACF reflects the diffusion of particles in the solution. For a polydisperse system, the contribution of all particles to the normalized $\operatorname{IACF}\left(\hat{g}_{I}^{\sigma}(\kappa, t)\right)$ may be averaged by a weighted integral in terms of the particle radius as follows: ${ }^{28}$

$$
\hat{g}_{I}^{\sigma}(\kappa, t)=1+C\left|\frac{\int_{0}^{\infty} \mathrm{d} a f_{\mathrm{psd}}(a) B^{2} \mathrm{e}^{\left(-D_{\mathrm{o}} \kappa^{2} t\right)}}{\int_{0}^{\infty} \mathrm{d} a f_{\mathrm{psd}}(a) B^{2}}\right|^{2}
$$

where the constant in the denominator normalizes the correlation function. The dynamic contrast factor $C$ in eqs 7 and 8, required to account for experimental limitations, allows the intercept of the normalized correlation function for $t \rightarrow 0$ to be smaller than 2 .

Computing particle shapes from light scattering experiments is difficult because this problem is ill-posed. Since the measured quantity in SLS is the intensity, the phase information is lost, something which hampers an inverse Fourier transformation. Different particle shapes may correspond to a single scattering intensity pattern that varies with the scattering angle $\theta$. Nevertheless, using, e.g., high resolution small angle scattering employing biomacromolecules, which generally lack shape heterogeneity, oversampling techniques can be used to obtain a low resolution particle shape. ${ }^{30}$ Another applicant is coherent X-ray diffraction to reveal the structure of nanocrystals. ${ }^{31}$

Regarding the ill-conditioning of the inversion problem, ${ }^{6}$ the shape of the particle size distribution should be known a priori for the analysis of the DLS correlation function. Generally, for unimodal polydisperse spherical colloids, the weight factor $f_{\mathrm{psd}}(a)$ is assumed to be a log-normal radius distribution, whose logarithm is normally distributed. Even if the shape is known, several combinations of mean particle radius $a_{\mathrm{AV}}$ and polydispersity $\sigma_{\mathrm{p}}$ can be described with the same correlation function. Therefore,

(27) Berne, B. J.; Pecora, R. Dynamic light scattering: with applications to chemistry, biology, and physics; Dover Publications: Mineola, NY, 2000.

(28) Dhont, J. K. G. An introduction to dynamics of colloids; Studies in interface science; Elsevier: New York, 1996; Vol. 2.

(29) Einstein, A. Ann. Phys. 1905, 322, 549-560.

(30) Petoukhov, M. V.; Konarev, P. V.; Kikhney, A. G.; Svergun, D. I. J. Appl. Crystallogr. 2007, 40, s223-s228.

(31) Pfeifer, M. A.; Williams, G. J.; Vartanyants, I. A.; Harder, R.; Robinson, I. K. Nature 2006, 442, 63-66. 
we generate a theoretical IACF using the discrete psd computed from the differential (envelope) vHW sedimentation coefficient distribution (including 99\% of each interference sedimentation boundary) obtained from the SV AUC interference optical data (Figure 3). Consequently, the integral in eq 8 is replaced by a summation over the $k$ distinct particle radii $a_{i}$ (see eqs 14 and 15) that comprise the discrete particle size distribution (histogram) $h_{\mathrm{psd}}\left(a_{i}\right)$ :

$$
\hat{g}_{I}^{\sigma}(\kappa, t)=1+C\left|\frac{\sum_{i=1}^{k} h_{\mathrm{psd}}\left(a_{i}\right) B^{2} \mathrm{e}^{\left(-D_{\mathrm{o}^{2}} t\right)}}{\sum_{i=1}^{k} h_{\mathrm{psd}}\left(a_{i}\right) B^{2}}\right| 2
$$

For comparison, we also use a continuous psd that is obtained by fitting:

$$
f_{\mathrm{psd}}(a)=A \exp [-\exp (-z)-z+1]
$$

to the discrete psd. In eq $10, z$ is equal to the following:

$$
z=\frac{\left(a-a_{\mathrm{c}}\right)}{w}
$$

$a$ is the radius, $a_{\mathrm{c}}, w$, and $A$ are, respectively, the center radius and the width and the amplitude of the psd. This psd (eq 10) is normalized such that:

$$
\int_{0}^{\infty} \mathrm{d} a f_{\mathrm{psd}}(a)=1
$$

Another approach is the frequently used second cumulant analysis method ${ }^{32}$ valid for spherical particles with a sufficiently narrow size distribution (often assumed to be log-normal) that is expanded to first-order in standard deviation. The drawback of this approach is that it restricts the polydispersity to a relative small number (i.e., $\leq 10 \%$ ). The second cumulant, which is directly related to the polydispersity, is very sensitive to the truncation of the correlation function. ${ }^{33}$ Moreover, the second cumulant analysis is valid only for the limit of $\kappa \rightarrow 0$.

\section{MATERIALS AND METHODS}

The colloidal dispersion employed here is commercially available as Bindzil 30/360 (Eka Chemicals AB Colloidal Silica Group, Bohus, Sweden). According to the product data sheet, it consists of discrete spherical amorphous silica particles with a uniform diameter of $9 \mathrm{~nm}$ in an aqueous solution. The colloidal silica dispersion is stabilized with a small amount of sodium hydroxide. Typical dispersion properties are shown in Table 1.

Since colloidal silica partly dissolves in aqueous solution due to the hydrolysis of $\mathrm{SiO}_{2}$ mediated by a base as $\mathrm{OH}^{-}$, the small Bindzil 30/360 are subjected to Ostwald-ripening,; ${ }^{34}$ i.e., larger

(32) Pecora, R. Dynamic light scattering: applications of photon correlation spectroscopy; Plenum: New York, 1985.

(33) Finsy, R.; de Groen, P.; Deriemaeker, L.; van Laethem, M. J. Chem. Phys. 1989, 91, 7374-7383.

(34) Everett, D. H. Basic principles of colloid science; Royal Society of Chemistry: London, 1988.
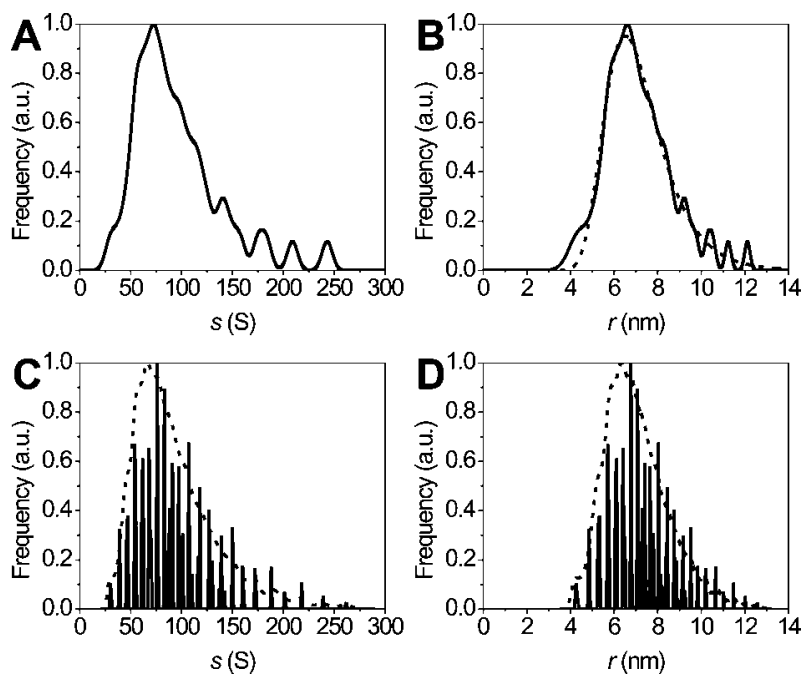

Figure 3. Apparent (i.e., not corrected for solvent density and viscosity) differential (envelope) sedimentation coefficient distribution $\left[(-)\right.$ graph A] as obtained from the enhanced vHW analysis ${ }^{17}$ (UltraScan) ${ }^{18}$ including $99 \%$ of each SV boundary. The corresponding psd (-) is shown in graph B. The sedimentation coefficient distribution (graph C) and the psd (graph D) obtained from a $c(s)$ analysis (sedfit) $^{20,21}$ with (- - -) and without (-) maximum entropy regularization $^{20}$ are equal to the enhanced $\mathrm{vHW}$ distributions. The continuous psd [(- - -), graph B], obtained by fitting eq 10 to the discrete differential psd, was used to fit the DLS correlation functions. All distributions were derived from SV AUC interference optical data.

\section{Table 1. Bindzil 30/360 Characteristics ${ }^{a}$}

$\begin{array}{ll}\mathrm{SiO}_{2} & 30.5 \% \mathrm{w} / \mathrm{w} \\ \text { particle diameter } & 9 \mathrm{~nm} \\ \text { titrable alkali as } \mathrm{Na}_{2} \mathrm{O} & 0.60 \% \mathrm{w} / \mathrm{w} \\ \mathrm{pH} & 10.0 \\ \text { dispersion density }^{b} & 1.218 \mathrm{~g} \mathrm{~cm}^{-3} \\ \text { dispersion viscosity }^{b} & 4 \mathrm{mPa} \mathrm{s} \\ \text { average specific surface area }^{-1} & 360 \mathrm{~m}^{2} \mathrm{~g}^{-1}\end{array}$

${ }^{a}$ Quantities according to the product data sheet (Eka Chemicals $\mathrm{AB}$ Colloidal Silica Group, Bohus, Sweden). ${ }^{b}$ Determined at $20^{\circ} \mathrm{C}$.

particles grow at the expense of small particles. The corresponding decrease of surface area in time is well-documented for aqueous silica sols. ${ }^{1}$

Analytical Ultracentrifugation. The stock dispersion as obtained from the manufacturer was diluted approximately 24 times ( $42 \mu \mathrm{L}$ stock dispersion $+958 \mu \mathrm{L}$ Milli-Q water) to achieve an optical density $\left(\mathrm{OD}_{229 \mathrm{~nm}}\right)$ that is in between 0.5 and 0.9 at 229 $\mathrm{nm}$. The latter wavelength is the shortest wavelength with an optimal signal-to-noise ratio when using the absorbance optics of the Beckman Coulter Optima XL-I AUC. The stock dispersion was diluted to avoid concentration effects, e.g. hydrodynamic and/or electrostatic interactions. According to Table 1, the SV AUC sample contains approximately $15.6 \mathrm{mg} \mathrm{mL}^{-1} \mathrm{SiO}_{2}$ spheres $(\mathrm{pH}$ $\approx 9.0$ ).

An estimate for the angular velocity from a Finite Element $t^{35}$ (FE) simulation (UltraScan $)^{18}$ was further optimized experimentally for a SV run employing the absorbance optics to resolve the whole range of $s$-values to obtain 45 scans that cover the solution column length. SV AUC experiments were conducted employing

(35) Demeler, B.; Saber, H. Biophys. J. 1998, 74, 444-454. 
$12 \mathrm{~mm}$ standard double sector charcoal-filled Epon centerpieces equipped with quartz (absorbance optics) or sapphire (interference optics) windows.

The sedimentation rate was determined with the absorbance and interference optics in two separate experiments on an identical sample $\left(\omega=18800 \mathrm{rpm}, T=20 \pm 0.1^{\circ} \mathrm{C}\right.$ ) to allow a comparison of data analysis results (see the Results and Discussion section).

SV data were analyzed with the enhanced $\mathrm{vHW}$ analysis ${ }^{17}$ and the $2 \mathrm{DSA}^{19}$ both implemented in UltraScan ${ }^{18}$ and with the $c(s)$ analysis (sedfit) ${ }^{20,21}$ methods. The latter was performed with and without maximum entropy regularization ${ }^{20}$ (confidence levels of 0.70 and 0.95 ).

The relative polydispersity $\left(\sigma_{\mathrm{p}}\right)$ in terms of the standard deviation $\left(\sigma_{\text {std }}\right)$ obtained from a sedimentation coefficient distribution reads as follows:

$$
\sigma_{\mathrm{p}}=\frac{\sigma_{\mathrm{std}}}{a_{\mathrm{AV}}} 100 \%
$$

The standard deviation follows from the square-root of the variance $V(a)$ :

$$
V(a)=\frac{1}{N} \sum_{i=1}^{k} n_{i}\left(a_{i}-a_{\mathrm{AV}}\right)^{2}
$$

with $N$ being the sum of frequencies $\left(n_{i}\right)$ of the $k$ distinct sedimentation coefficients and $a_{\mathrm{AV}}$ being the average (arithmetic mean) radius that follows:

$$
a_{\mathrm{AV}}=\frac{\sum_{i=1}^{k} n_{i} a_{i}}{\sum_{i=1}^{k} n_{i}}
$$

$n_{i}$ is directly proportional to the concentration $c_{i}$ of solute(s) or colloid(s) $i$. For a vHW analysis, $n_{i}$ is proportional to the amount of linear extrapolations for which the extrapolated intercept at $t$ $=\infty$ is in between two consecutive sedimentation coefficients $s_{i}$ and $s_{i+1}$.

Specific Particle Volume from SV Interference Data. The specific particle volume can be obtained from a single analytical ultracentrifugation sedimentation velocity run using the interference optics (Beckman Coulter Optima XL-I AUC), provided that all species have the same refractive index, which can be obtained from literature (e.g., ref 36), and that the initial sample loading concentration is known. The key parameter is the total fringe displacement, $Y_{\text {tot }}$, via ${ }^{37}$ the following:

$$
Y_{\mathrm{tot}}=\sum\left(c_{i} \frac{\partial n}{\partial c_{i}}\right) \frac{l}{\lambda}
$$

with $c_{i}$ being the concentration of component $i, l$ the cell path

(36) Lide, D. R. CRC handbook of chemistry and physics: a ready-reference book of chemical and physical data, 81st ed.; CRC: Boca Raton, FL, 2000.

(37) Laue, T. M. Choosing Which Optical System of the Optimal XL-I Analytical Ultracentrifuge to Use; Technical Report A-1821A, Beckman Coulter: Pala Alto, CA, 1996. length $(12 \mathrm{~mm})$, and $\lambda$ the optical wavelength $(675 \mathrm{~nm}) . Y_{\text {tot }}$ for the total initial loading concentration can be determined from a sedimentation velocity run by extrapolating the fitted plateau concentrations (fringe displacement) of each sedimentation velocity scan included in the analysis with a polynomial to the time at which the rotor started to accelerate $t=0$. If the dispersion contains one species only, $\partial n / \partial c_{i}$ in eq 16 can be taken out of the summation and the specific refractive index can be calculated by substitution of $Y_{\text {tot }}, \Sigma c_{i}=c_{\text {tot }}, l$, and $\lambda$ into eq 16 . The specific particle volume then follows from the differential refractive index $\partial n / \partial c_{\text {tot }}$ (i.e., specific index of refraction) by substitution of the particle $\left(n_{\mathrm{p}}\right)$ and solvent $\left(n_{\mathrm{s}}\right.$, corrected for added salt concentration) refractive indices in the following:

$$
\frac{\partial n}{\partial c_{\text {tot }}}=\frac{n_{\mathrm{p}}-n_{\mathrm{s}}}{\rho_{\mathrm{p}}}
$$

The total fringe displacement from the exponential fit (enhanced vHW analysis, UltraScan) for the initial loading concentration of $c_{\text {tot }}=15.6 \mathrm{mg} \mathrm{mL}^{-1}$ (using the weight concentration from Table 1 and accounting for the dilution) is $Y_{\text {tot }}=18.8$. Substitution of $Y_{\text {tot }}, c_{\text {tot }}$, the laser's wavelength $\lambda=675 \mathrm{~nm}$, and the centerpiece path length $l=12 \mathrm{~mm}$ in eq 16 yields a differential refractive index of $\partial n / \partial c_{\text {tot }}=6.78 \times 10^{-5} \mathrm{~mL} \mathrm{mg}^{-1}$. Next, the specific Bindzil $30 / 360$ particle volume, $\bar{v}_{\mathrm{p}}=1 / \rho_{\mathrm{p}}=0.57 \mathrm{~mL} \mathrm{~g}^{-1}$, follows from eq 17 upon substitution of $n_{\mathrm{s}}=1.33$ and $n_{\mathrm{Si}_{2}}=1.45$ (amorphous $\mathrm{SiO}_{2}$ ) from ref 36.

Dynamic Light Scattering. The dispersion available for DLS measurements was a silica sol that had been dialyzed extensively in a previous study ${ }^{22}$ against ethanol. This dialysis was needed for a surface functionalization, which cannot be done in water. ${ }^{22}$ Since this dialysis transfer to ethanol does not affect the colloidal stability of the silica particles, ${ }^{22}$ we can compare the results from DLS and SV AUC measurements on aqueous silica sols.

Correlation functions at six equally spaced scattering angles, ranging from $\theta=35$ to $140^{\circ}$, were determined for a diluted Bindzil 30/360 sample employing a Krypton-ion laser (Spectra Physics, type 2025-11) as the incident light beam for which the in vacuo wavelength is $\lambda_{0}=647.1 \mathrm{~nm}$. The correlation functions were recorded with a multiple tau digital correlator (ALV, type 6010/ 160). In the detector (ALV/SO-SPID), the beam passes a splitter and is directed into two single-photon photomultiplier tubes (PMT's), from which a pseudocorrelation function was produced to suppress the dark current generated by after-pulsing and thermal noise. Since the scattering power of the Bindzil 30/360 particles is relatively low due to the small particle dimensions, the correlation function at each scattering angle was measured during $2 \mathrm{~h}$, to increase the signal-to-noise ratio and, consequently, to obtain smooth correlation functions.

For the theoretical correlation functions, the hydrodynamic radii required to compute translational diffusion coefficients in eqs 8 and 9 via the Stokes-Einstein ${ }^{23,29}$ relation, are assumed to equal the bare particle radii.

\section{RESULTS AND DISCUSSION}

As already addressed, the size and shape of Bindzil 30/360 particles cannot be precisely determined from TEM micrographs 


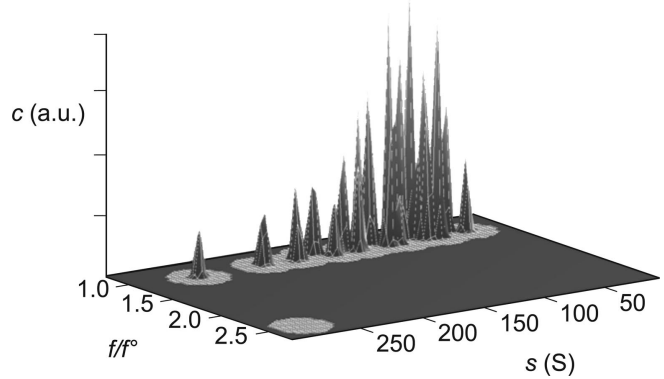

Figure 4. Three-dimensional plot of particle concentration $(c$ in arbitrary units) vs the frictional ratio $\left(f / f^{\circ}\right)$ and sedimentation coefficient $(s)$ as obtained from a 2-dimensional spectrum analysis (UltraScan) ${ }^{18}$ on the SV AUC interference optical data. All particles are spherical, i.e. the frictional ratio for all silica nanoparticles within this large sedimentation coefficient range is equal or close to $f / f^{\circ}=1.0$, assuring that the conversion of the vHW differential sedimentation coefficient distribution into a radius distribution via eq 3 is appropriate.

(Figure 1), since the small silica particles easily melt in the electron beam. The average particle size and shape, as well as their distributions, however, can be assessed straightforward by a single SV AUC experiment. In the following AUC Experiments section, the results from SV AUC experiments are discussed and compared, in the SV AUC and DLS Comparison section, with the DLS correlation functions.

AUC Experiments. The sedimentation coefficients distribution, corrected for diffusion, obtained from an enhanced $\mathrm{vHW}$ analysis, shows that the Bindzil 30/360 particles exhibit size and/ or shape polydispersity (graph $\mathrm{A}$ and $\mathrm{C}$ in Figure 3 ). The sedimentation coefficients of these particles range from approximately $20-250 \mathrm{~S}$.

The range and overall shape of the vHW differential and the 2DSA sedimentation coefficient distributions shown in Figures 3 (graph A) and 4 are almost identical. Furthermore, Figure 4 clearly shows that the particle size is very heterogeneous, whereas the particle shape appears to be uniform; i.e. the frictional ratio for all silica nanoparticles is $f / f^{\circ}=1$, indicating that all particles are spherical. The sedimentation coefficient distribution from the 2DSA may give the impression that the sample contains discrete species. But since we did not apply any regularization or solute coalescing, the result obtained from the 2DSA will comprise the fewest number of solutes with which the experimental data can still be described reasonably well.

Having determined the uniform spherical shape of the Bindzil $30 / 360$ particles, the conversion of the sedimentation coefficient distribution into a radius distribution is straightforward. This conversion can be done as follows.

First, the specific particle volume (or partial specific volume) should be available and may be obtained independently or from the same experimental SV AUC interference optical data (single run) that was acquired to determine the sedimentation coefficient and particle shape distribution. Here, we use a specific particle volume of $\bar{v}_{\mathrm{p}}=1 / \rho_{\mathrm{p}}=0.57 \mathrm{~mL} \mathrm{~g}^{-1}$, which is determined as described in the Specific Particle Volume from SV Interference Data section.

Second, the solvent viscosity and density are required. The aqueous SV AUC sample solution contains approximately $77 \mathrm{mM}$ $\mathrm{NaOH}$. Therefore, the density and viscosity of the solution in which the silica particles sediment are, respectively, $\rho_{\mathrm{s}}=1.0019$
Table 2. vHW Particle Size Distributions ${ }^{a}$

$\begin{array}{lcc} & \text { absorbance }^{b} & \text { interference }^{c} \\ a_{\mathrm{AV}}(\mathrm{nm})^{d} & 7.7 & 7.4 \\ V(a)\left(\mathrm{nm}^{2}\right)^{e} & 1.6 & 1.2 \\ \sigma_{\mathrm{std}}(\mathrm{nm})^{f} & 1.3 & 1.1 \\ \sigma_{\mathrm{p}}(\%)^{g} & 16.3 & 14.8\end{array}$

${ }^{a}$ vHW analysis results including $90 \%$ of each boundary (Figure 3). ${ }^{b}$ Results for SV AUC absorbance data. ${ }^{c}$ Results for SV AUC interference data. ${ }^{d}$ Average particle radius (eq 15). ${ }^{e}$ Variance (eq 14). ${ }^{f}$ Absolute standard deviation. ${ }^{g}$ Size polydispersity (eq 13).

\section{Table 3. vHW, 2DSA, and c(s) Results ${ }^{a}$}

$\begin{array}{lrrrr} & \mathrm{vHW}^{b} & { }^{2} \mathrm{DSA}^{c} & c(s)^{d} & c(s)^{e} \\ a_{\mathrm{AV}}(\mathrm{nm})^{f} & 7.4 & 7.5 & 7.3 & 7.3 \\ V(a)\left(\mathrm{nm}^{2}\right)^{g} & 2.7 & 2.6 & 2.4 & 2.4 \\ \sigma_{\mathrm{std}}(\mathrm{nm})^{h} & 1.6 & 1.6 & 1.5 & 1.5 \\ \sigma_{\mathrm{p}}(\%)^{i} & 22.0 & 21.4 & 21.0 & 21.2\end{array}$

${ }^{a}$ All values are obtained from the SV AUC interference optical data. ${ }^{b}$ van Holde-Weischet analysis results including $99 \%$ of each boundary. ${ }^{c}$ Two-dimensional spectrum analysis (Figure 4). ${ }^{d}$ Continuous distribution of sedimentation coefficients analysis. ${ }^{20,21} e c(s)$ analysis ${ }^{20,21}$ with maximum entropy regularization. ${ }^{20} f$ Average particle radius (eq 15). ${ }^{g}$ Variance (eq 14). ${ }^{h}$ Absolute standard deviation. ${ }^{i}$ Size polydispersity (eq 13).

$\mathrm{g} \mathrm{mL} \mathrm{m}^{-1}$ and $\eta_{\mathrm{s}}=1.0125 \mathrm{mPa} \mathrm{s}$ (computed with the buffer correction module implemented in UltraScan ${ }^{18}$ ).

Third, substituting all required parameters previously addressed in eq 3 yields the radius distributions (Figure 3, graphs $\mathrm{B}$ and $\mathrm{D})$. The average (arithmetic mean) radius, the variance, the standard deviation, and the relative polydispersity can be computed from the psd via eqs $13-15$.

Clearly, the Bindzil 30/360 particles exhibit a significant size polydispersity (Tables 2 and 3) though for silica colloids of this small dimension the polydispersity is actually fairly small. The SV AUC results from the interference and absorbance optics data including $90 \%$ of each sedimentation boundary on the size determination are similar (Table 2). Identical results would ensure that using a single extinction coefficient for "all" species contained in the dispersion is appropriate, in contrast to the case of polydisperse colloidal dispersions that cover a very broad size range. For the latter system, the fraction of large particles may be overestimated from absorbance data, since the scattered intensity scales with the particle volume squared. The small deviation of the average size and relative polydipersity (Table 2) is likely due to this fact.

However, the almost quantitative agreement of the psd from the SV AUC absorbance optical data with the interference optical data is remarkably good, considering that absorbance data generally have a much lower signal-to-noise ratio compared to interference data, in particular for particles that have a relative large refractive index difference and a relatively small extinction coefficient. For colloids that have a much larger refractive index than the dispersion medium, a steep concentration gradient, and, consequently, a steep gradient in refractive index, develops during the early stage of the sedimentation velocity experiment. Such gradients result in the reflection of light that appears as a peak in the absorbance optical data. These peaks are equivalent to the peaks observed for menisci using the absorbance optical system. 
This effect decreases during the time course of the sedimentation velocity run because of diffusion and radial dilution. Together with the time invariant noise, the reflection of light contributes to the deviations previously addressed. Therefore, in addition to the higher signal-to-noise ratio for the interference data, a psd of silica colloids from interference optical data will, in general, be more accurate than from absorbance optical data.

The effect of excluding $5 \%$ of each sedimentation boundary at the top and the bottom in a vHW analysis is evident from Tables 2 and 3 . The smallest and largest colloids are not covered by the psd if upper and lower portions of sedimentation velocity boundaries are clipped off. Generally, a small portion of each sedimentation boundary is excluded in a vHW analysis to maintain the correlation of divisions. This exclusion may result in an apparent decreased polydispersity. Here, the data quality is sufficient to include $99 \%$ of each sedimentation velocity boundary (this is usually not the case for SV AUC absorbance optical data).

It can be seen from Figure 3 that the enhanced vHW (including $99 \%$ of each SV boundary) and the $c(s)$ (whole boundary fitting routine) sedimentation coefficient distributions and psd's, derived from the same SV AUC interference optical data, are almost equal. Consequently, the average radius, the variance, and the relative polydispersity obtained from these sedimentation coefficient distributions are not significantly different. Furthermore, the $c(s)$ analysis (sedfit) ${ }^{20,21}$ was performed with and without maximum entropy regularization. ${ }^{20}$ In contrast to what is reported for the size distribution of a multiprotein sample ${ }^{38}$ we show here that the model independent sedimentation coefficient distribution obtained via a vHW analysis yields the same results as the $c(s)$ method. Since the $c(s)$ analysis method assumes that all particles have a uniform shape (single frictional ratio for all species), this equality is due to the homogeneous shape of the silica colloids employed here. However, the agreement is also due to the large spread in $s$-values and in particular to the fact that the "true" psd of Bindzil is nearly continuous. In this case, for a sufficiently large amount of divisions, the resolution of the vHW analysis is just as good as the resolving ability of whole boundary fitting methods. The only effect of regularization on the sedimentation coefficient distribution and the corresponding psd is smoothing. The $s$ and $a$ distributions do not change, even if the confidence interval is varied from 0.70 to 0.95 . Since regularization has no effect and taking into account that the $c(s)$ constrains the frictional ratio to a single value, the enhanced vHW analysis is the preferred method to assess the polydispersity of colloids. Moreover, the vHW analysis is, in principle, model independent and the determination of a psd with this analysis is therefore straightforward.

The results shown in Table 3 conform well to particle dimensions obtained from TEM (image A in Figure 1). But are the results obtained from SV AUC consistent with the experimental DLS correlation functions?

SV AUC and DLS Comparison. The consistency of SV AUC and TEM particle dimensions can be verified by an overlay of the theoretical polydisperse intensity autocorrelation functions computed via eqs 8 and 9 for, respectively, the continuous and discrete psd and the experimental IACF (Figure 5). The theoretical correlation functions closely follow the experimental IACF. The

(38) Schuck, P.; Perugini, M. A.; Gonzales, N. R.; Hewlett, G. J.; Schubert, D. Biophys. J. 2002, 82, 1096-1111.

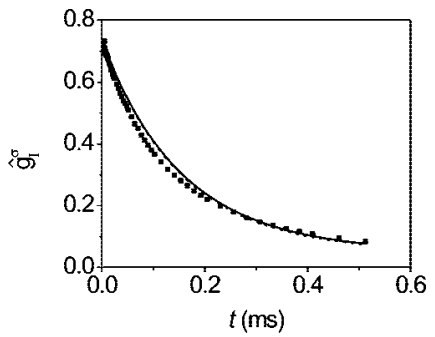

Figure 5. Theoretical polydisperse intensity autocorrelation functions calculated via eqs 8 and 9 for, respectively, the continuous $(-)$ and discrete (- - ) psd. The experimental IACF $\left(\square, \theta=56^{\circ}\right.$ or $\kappa=1.24$ $\times 10^{7} \mathrm{~m}^{-1}$ ) follows the theoretical IACF's closely. The discrete psd obtained from the enhanced $\mathrm{vHW}$ analysis (including $99 \%$ of each sedimentation boundary) results in the same IACF as the continuous psd, which is a fit of eq 10 to the discrete psd.

root-mean-square (rms) deviations of the theoretical and experimental correlation functions reveal that the predicted correlation function using the discrete and the continuous psd are not significantly different.

Confirming our expectation, the second cumulant analysis method yields for the Bindzil 30/360 particles an apparent polydispersity of $30 \%$ or larger, which is much larger than all other results obtained here, implying that this apparent polydispersity is physically not relevant. Another approach is to determine the theoretical correlation function for spherical particles with a lognormal size distribution. As mentioned earlier, several combinations of a mean radius and polydispersity result in the same correlation function. Defining the mean radius as obtained from the enhanced vHW analysis (Table 3 ) and using the polydispersity as a fit parameter, the rms of the residuals of the theoretical and experimental correlation can be minimized. In the limit of $\kappa \rightarrow 0$, the resulting polydispersity is $23 \%$ and agrees well with the polydispersities determined from the SV AUC optical interference data (Table 3). However, rms deviations of the IACF generated using the vHW psd's and the experimental correlation function are significantly smaller (30\%) compared to the IACF for a lognormal psd.

\section{CONCLUSIONS}

We have shown that sedimentation velocity analytical ultracentrifugation (SV AUC) is very suited for the in situ determination of average sizes and shapes of small colloids in solution. A single SV AUC experiment already contains sufficient information to determine the particle size and shape distributions, as well as the specific particle volume. From the psd, all relevant quantities such as the average radius, variance, standard deviation, and relative polydispersity can be computed.

Whole boundary fitting methods and in particular the continuous distribution of sedimentation coefficients, $c(s)$, do not offer any advantage compared to the model independent enhanced van Holde-Weischet analysis. Additionally, it should be noted that the procedure reported here including sample preparation, to eventually obtain the psd and all relevant statistical parameters, can in principle be conducted within a single day. Our method to determine the average size and polydispersity from SV AUC experiments may also be applied to other particle shapes to obtain an apparent polydispersity, for which the concept of equivalent sphere radii may be introduced. 
For colloidal silica dispersions an AUC equipped with interference optics yields data with a higher signal-to-noise ratio compared to absorbance optics and in addition provides information on the specific particle volume. Therefore, using the interference optics for dispersed particles that have a relatively large refractive index compared to the solvent and that absorb only weakly in the $\mathrm{UV}$-vis region yields more reliable results.

The discrete particle size distribution obtained from SV AUC yields an intensity autocorrelation function that agrees much better with the measured correlation function from DLS than a fit with a log-normal size distribution. Additionally, we found that the second cumulant analysis fails and is indeed not valid for the relatively large polydispersity of the silica particles employed here.

\section{ACKNOWLEDGMENT}

We thank E. Maria Claesson for providing TEM images and DLS data and Stefano Sacanna for helpful discussions. Ben H. Erné and Kees C. G. de Kruif are thanked for helpful discussions and suggestions. Eka Chemicals AB Colloidal Silica Group (Bohus, Sweden) is thanked for providing the Bindzil 30/360 dispersion.

Received for review July 24, 2008. Accepted August 26, 2008.

AC801556T 